Itumeleng B. Setshedi, Andreas Lemmerer and Mark G. Smith*

\title{
The crystal structure of $(E)-N^{\prime}$-(butan-2-ylidene) isonicotinohydrazide 0.5 hydrate $\mathrm{C}_{10} \mathrm{H}_{13} \mathrm{~N}_{3} \mathrm{O}$. $0.5 \mathrm{H}_{2} \mathrm{O}$
}

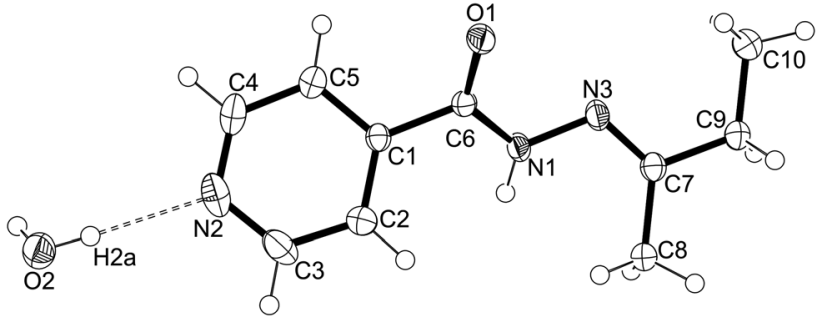

https://doi.org/10.1515/ncrs-2021-0311 Received July 30, 2021; accepted August 16, 2021; published online August 31, 2021

Table 1: Data collection and handling.

Crystal:

Size:

Wavelength:

$\mu$ :

Diffractometer, scan mode:

$\theta_{\text {max }}$, completeness:

$N(h k l)_{\text {measured }}, N(h k l)_{\text {unique }}, R_{\text {int }}$ : Criterion for $l_{\text {obs }}, N(h k l)_{\text {gt }}$ : $N(\text { param })_{\text {refined: }}$ Programs:

\author{
Colourless block \\ $0.91 \times 0.79 \times 0.14 \mathrm{~mm}$

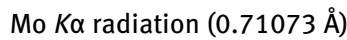 \\ $0.09 \mathrm{~mm}^{-1}$ \\ Bruker D8 Venture Photon, $\omega$ \\ $28.0^{\circ},>99 \%$ \\ $58009,2526,0.040$ \\ $I_{\text {obs }}>2 \sigma\left(I_{\text {obs }}\right), 2474$ \\ 138 \\ Bruker [1], SHELX [2], WinGX [3], \\ PLATON [4], ORTEP-III [5]
}

\begin{abstract}
$\mathrm{C}_{10} \mathrm{H}_{13} \mathrm{~N}_{3} \mathrm{O} \cdot 0.5 \mathrm{H}_{2} \mathrm{O}$, orthorhombic, $A b a 2$ (no. 41), $a=19.9676(19) \AA, b=8.4674(7) \AA, c=12.4082(10) \AA$, $V=2097.9(3) \AA^{3}, Z=8, R_{g t}(F)=0.0299, w R_{r e f}\left(F^{2}\right)=0.0849$, $T=173 \mathrm{~K}$.
\end{abstract}

\section{CCDC no.: 2086756}

The asymmetric unit of the title structure is shown in the figure. Table 1 contains crystallographic data and Table 2 contains the list of the atoms including atomic coordinates and displacement parameters.

\section{Source of material}

All reagents used were purchased and used without further purification. Isonicotinic acid hydrazide (isoniazid) (1.0 g; $7.29 \mathrm{mmol}$ ) and $15.0 \mathrm{~mL}$ of 2-butanone were added to a

*Corresponding author: Mark G. Smith, Chemistry Department, University of South Africa, Unisa Science Campus, 28 Pioneer Avenue, Florida, Roodepoort, Gauteng, South Africa,

E-mail: smithm2@unisa.ac.za. https://orcid.org/0000-0003-25532540

Itumeleng B. Setshedi, Department of Life Science, University of South Africa, Unisa Science Campus, 28 Pioneer Avenue, Florida, Roodepoort, Gauteng, South Africa. https://orcid.org/0000-00018865-4735

Andreas Lemmerer, School of Chemistry, Molecular Sciences Institute, University of the Witwatersrand, Johannesburg, Gauteng, South Africa. https://orcid.org/0000-0003-1569-2831
$25 \mathrm{~mL}$ stainless steel jar containing $2 \times 7 \mathrm{~mm}$ stainless steel balls. The jar was tightly sealed and the contents were shaken for $30 \mathrm{~min}$ at $25 \mathrm{~Hz}$ using the Retsch Mixer Mill MM 200. The mixture was then transferred into a $50 \mathrm{ml}$ amber Schott bottle and left slightly open to allow slow evaporation at room temperature. Colourless block crystals were observed after three days.

\section{Experimental details}

The collection method involved $\omega$-scans of width $0.5^{\circ}$. Data reduction was carried out using SAINT-Plus version 6.02.6 software, and SADABS was used to process empirical absorption corrections [1]. Hydrogen atoms were allowed to ride on their respective parent atoms with $\mathrm{d}(\mathrm{C}-\mathrm{H})=0.95$ [2]. Hydrogen atoms were positioned geometrically $\AA$ and $U_{i s o}(\mathrm{H})=1.2 U_{e q}(\mathrm{C})$. Diagrams and publication material were generated using WinGX [3], PLATON [4] and Ortep [5, 6].

\section{Comment}

Isoniazid, a synthetic derivative of nicotinic acid [7] has been used as a frontline drug for the treatment of Tuberculosis (TB) for decades [8]. Tuberculosis-causing mycobacteria have developed resistance against this drug, thus there is an urgent need for novel and effective drug design and 
Table 2: Fractional atomic coordinates and isotropic or equivalent isotropic displacement parameters $\left(\AA^{2}\right)$.

\begin{tabular}{lrrrr}
\hline Atom & $\boldsymbol{x}$ & $\boldsymbol{y}$ & $\boldsymbol{z}$ & $\boldsymbol{U}_{\text {iso }}{ }^{*} \boldsymbol{U}_{\text {eq }}$ \\
\hline C1 & $0.33289(7)$ & $0.36429(17)$ & $0.48805(12)$ & $0.0191(3)$ \\
C2 & $0.30391(9)$ & $0.2671(2)$ & $0.41159(14)$ & $0.0277(3)$ \\
H2 & 0.2566 & 0.2608 & 0.4052 & $0.033^{*}$ \\
C3 & $0.34505(11)$ & $0.1786(2)$ & $0.34421(16)$ & $0.0359(4)$ \\
H3 & 0.3247 & 0.1136 & 0.2911 & $0.043^{*}$ \\
C4 & $0.43912(9)$ & $0.2735(2)$ & $0.42588(16)$ & $0.0329(4)$ \\
H4 & 0.4865 & 0.2746 & 0.4321 & $0.039^{*}$ \\
C5 & $0.40252(8)$ & $0.3686(2)$ & $0.49557(14)$ & $0.0247(3)$ \\
H5 & 0.4243 & 0.4344 & 0.5468 & $0.03^{*}$ \\
C6 & $0.29301(7)$ & $0.47594(17)$ & $0.55704(12)$ & $0.0196(3)$ \\
C7 & $0.13160(8)$ & $0.50920(19)$ & $0.65346(13)$ & $0.0211(3)$ \\
C8 & $0.09319(8)$ & $0.3983(2)$ & $0.58217(16)$ & $0.0295(4)$ \\
H8A & 0.118 & 0.3815 & 0.515 & $0.044^{*}$ \\
H8B & 0.0872 & 0.297 & 0.6192 & $0.044^{*}$ \\
H8C & 0.0493 & 0.4439 & 0.5657 & $0.044^{*}$ \\
C9 & $0.09022(8)$ & $0.6141(2)$ & $0.72541(13)$ & $0.0235(3)$ \\
H9A & 0.0624 & 0.5461 & 0.7725 & $0.028^{*}$ \\
H9B & 0.0593 & 0.6761 & 0.6795 & $0.028^{*}$ \\
C10 & $0.12885(9)$ & $0.7277(2)$ & $0.79610(16)$ & $0.0309(4)$ \\
H10A & 0.1608 & 0.7871 & 0.7519 & $0.046^{*}$ \\
H10B & 0.1532 & 0.6684 & 0.8515 & $0.046^{*}$ \\
H10C & 0.0976 & 0.8011 & 0.8307 & $0.046^{*}$ \\
N1 & $0.23250(6)$ & $0.42502(15)$ & $0.58957(12)$ & $0.0237(3)$ \\
H1A & 0.2167 & 0.3325 & 0.5695 & $0.028^{*}$ \\
N2 & $0.41190(9)$ & $0.1807(2)$ & $0.35055(14)$ & $0.0373(4)$ \\
N3 & $0.19548(7)$ & $0.52638(16)$ & $0.65723(12)$ & $0.0238(3)$ \\
O1 & $0.31550(6)$ & $0.60805(13)$ & $0.57675(11)$ & $0.0276(3)$ \\
O2 & 0.5 & 0 & $0.20287(16)$ & $0.0330(4)$ \\
H2A & $0.4739(15)$ & $0.050(3)$ & $0.244(3)$ & $0.059(9)^{*}$ \\
\hline & & & &
\end{tabular}

development. Modifying existing drugs to target resistant organisms intend to mitigate the deactivation process by the mycobacteria [9]. The crystal structure being reported in this article represents a covalent modification of isoniazid. The compound crystallizes in the orthorhombic Aba2 space group. The asymmetric unit shown in the figure is a hemihydrate, containing one organic target molecule and half a water molecule. The water sits in a special position in the unit cell, with a twofold rotation axis passing through the oxygen atom of the water molecule. Bond lengths and angles are all in the expected ranges [10,11]. The crystal packing contains bilayers of $(E)$ - $N^{\prime}$-(butan-2-ylidene)isonicotinohydrazide alternating with layers of water molecules. Each carbohydrazide moiety is hydrogen bonded by a $\mathrm{O} 2-\mathrm{H} 2 \mathrm{a} \cdots \mathrm{N} 2$ hydrogen bond to a water molecule to form the hemihydrate. Each carbohydrazide moiety is also hydrogen bonded via its $\mathrm{N} 1-\mathrm{H}$ donor to the carbonyl oxygen (01) acceptor of an adjacent carbohydrazide moiety. The water present in the crystal is generated as a byproduct of the Schiff base reaction between isoniazid and butanone and is not added to the reaction mixture.

Author contributions: All the authors have accepted responsibility for the entire content of this submitted manuscript and approved submission.

Research funding: This work was supported by the National Research Foundation (NRF) Thuthuka Grant Number 118127 as well as Thuthuka Grant Number 117850. Conflict of interest statement: The authors declare no conflicts of interest regarding this article.

\section{References}

1. Bruker. SAINT-Plus and SADABS; Bruker AXS Inc.: Madison, Wisconsin. USA, 2004.

2. Sheldrick G. M. A short history of SHELX. Acta Crystallogr. 2008, A64, 112-122.

3. Farrugia L. J. WinGX suite for small-molecule single-crystal crystallography. J. Appl. Crystallogr. 1999, 32, 837-838.

4. Spek A. L. Structure validation in chemical crystallography. Acta Crystallogr. 2009, D65, 148-155.

5. Burnett M. N., Johnson C. K. ORTEPIII Report ORNL-6895; Oak Ridge National Laboratory: Tennessee, USA, 1996.

6. Farrugia L. J. WinGX and ORTEP for Windows: an update. J. Appl. Crystallogr. 2012, 45, 849-854.

7. Pubchem. Isoniazid. Open chemistry database, 2018, 1-75. Available from https://pubchem.ncbi.nml.nih.gov/compound/ isoniazid\#section=Top.

8. Setshedi I. B., Smith M. G. The crystal structure of the co-crystal: 2-hydroxybenzoic acid $N^{\prime}$-(butan-2-ylidene)pyridine-4carbohydrazide, $\mathrm{C}_{10} \mathrm{H}_{13} \mathrm{~N}_{3} \mathrm{O} \cdot \mathrm{C}_{7} \mathrm{H}_{6} \mathrm{O}_{3}$. Z. Kristallogr. N. Cryst. Struct. 2021, 236, 1093-1095.

9. Hu Y.-Q., Zhang S., Zhao F., Gao C., Feng L.-S., Lv Z.-S. Isoniazid derivatives and their anti-tubercular activity. Eur. J. Med. Chem. 2017, 133, 255-267.

10. Madeley L. G., Levendis D. C., Lemmerer A. Covalent- assisted supra molecular synthesis: the effect of hydrogen bonding in cocrystals of 4-tert-butyl benzoic acid with isoniazid and its derivatized forms. Acta Crystallogr. 2019, C75, 200-207.

11. Lemmerer A., Bernstein J., Kahlenberg V. Covalent assistance in supramolecular synthesis: in situ modification and masking of the hydrogen bonding functionality of the supramolecular reagent isoniazid in co-crystals. CrystEngComm 2011, 13, 5692-5708. 